\title{
BBN, Neutrinos and Nuclear Astrophysics
}

\author{
Carlo Gustavino \\ INFN ROMA \\ E-mail: carlo.gustavino@romal.infn.it
}

Big Bang Nucleosynthesis (BBN) theory describes the formation of light isotopes such as $\mathrm{D},{ }^{3} \mathrm{He}$, ${ }^{4} \mathrm{He},{ }^{6} \mathrm{Li}$ and ${ }^{7} \mathrm{Li}$ in the first minutes of cosmic time. Their abundance only depends on the baryon density, on particle physics and on nuclear astrophysics, through the competition between the universal expansion rate and the yields of the relevant nuclear reactions. As the expansion rate increases with the number of neutrino families (and any other relativistic species), the comparison between computed and observed abundances of light isotopes allows to constrain the number of neutrinos species, provided that the knowledge of the relevant nuclear processes is accurate enough. Starting from the present uncertainty of the relevant parameters (i.e. baryon density, observed abundance of isotopes and BBN nuclear cross sections), it will be shown that a renewed study of several nuclear reactions, possibly with existing or proposed underground accelerator facilities, is essential to improve the accuracy of computed abundances of light isotopes, providing the BBN theory a powerful probe of particle physics beyond the standard model. In particular, it will be shown that the accurate measurement of the $D(p, \gamma)^{3} \mathrm{He}$ reaction at BBN energies (40$400 \mathrm{keV}$ ), is of primary importance to constrain the number of active neutrinos and/or the lepton degeneracy in the neutrino sector.

52 International Winter Meeting on Nuclear Physics - Bormio 2014,

27-31 January 2014

Bormio, Italy 


\section{Big Bang Nucleosynthesis}

In the standard cosmology the expansion rate of the universe is governed by the Freidmann equation:

$$
H^{2}=\frac{8 \pi}{3} G \rho
$$

Were $H$ is the Hubble parameter, $G$ is the Newton's gravitational constant and $\rho$ is the energy density which, in the early Universe, is dominated by the "radiation", i.e. the contributions from massless or extremely relativistic particles. The only known relativistic particle at the Big Bang Nucleosynthesis (BBN) epoch are the photons (with $g_{\gamma}=2$ degrees of freedom or helicities) and the three neutrino families (with $g_{v}=2$, taking into account left-handed neutrinos and right-handed antineutrinos). Therefore, the abundance of primordial isotopes only depends on particle physics, on the baryon density $\Omega_{b}$ (or equivalently on the baryon-to-photon ratio $\eta$ ) and on the nuclear processes relevant in the BBN reaction chain, through the competition between the universal expansion rate and the yields of the relevant nuclear reactions.

As shown in figure 1, the nucleosynthesis begins with the formation of deuterium in the pro-

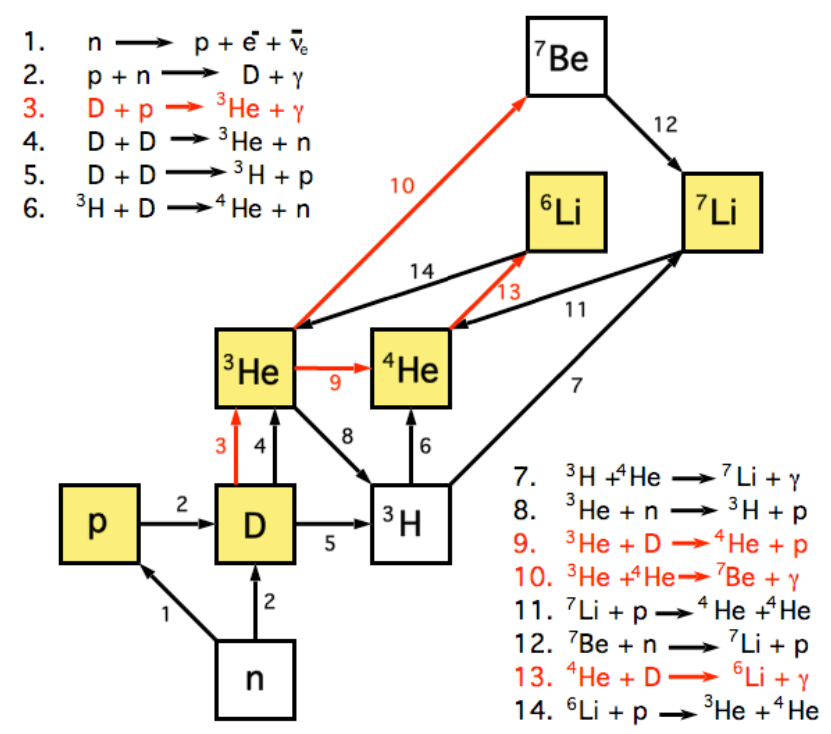

Figure 1: Leading processes of Big Bang Nucleosynthesis. Yellow boxes mark stable isotopes.

cess $p(n, \gamma) D$. Because of the large density of photons $\left(\eta \simeq 6 \cdot 10^{-10}\right)$, the photo-dissociation delays the production of deuterium well after $T$ drops below the binding energy of deuterium $(Q=2.23 \mathrm{MeV})$. Nearly all the free neutrons end up bound in the most stable light element ${ }^{4} \mathrm{He}$. Therefore, the abundance of ${ }^{4} \mathrm{He}$ mostly depends on the fraction of free neutrons available, and its uncertainty is almost entirely due to the neutron lifetime error. Heavier nuclei do not form in any significant quantity both because of the absence of stable nuclei with mass number 5 or 8 (which impedes nucleosynthesis via ${ }^{4} \mathrm{He}+n,{ }^{4} \mathrm{He}+p$ or ${ }^{4} \mathrm{He}+{ }^{4} \mathrm{He}$ reactions) and of the large Coulomb barriers for reactions such as the ${ }^{3} \mathrm{H}\left({ }^{4} \mathrm{He}, \gamma\right){ }^{7} \mathrm{Li}$ and $\mathrm{D}\left({ }^{4} \mathrm{He}, \gamma\right){ }^{6} \mathrm{Li}$. The primordial abundances of all the other stable isotopes, namely $(\mathrm{D} / \mathrm{H})_{B B N},\left({ }^{3} \mathrm{He} / \mathrm{H}\right)_{B B N},\left({ }^{7} \mathrm{Li} / \mathrm{H}\right)_{B B N}$ and $\left({ }^{6} \mathrm{Li} / \mathrm{H}\right)_{B B N}$ are 
very sensitive to reaction yields of the relevant nuclear processes. The uncertainty of $(D / H)_{B B N}$ abundance is presently dominated by the $D(p, \gamma)^{3} \mathrm{He}$ reaction, because the competing deuteriumburning channels $D\left({ }^{2} \mathrm{H}, p\right)^{3} \mathrm{H}$ and the $D\left({ }^{2} \mathrm{H}, n\right)^{3} \mathrm{He}$ have recently been studied with high accuracy $[1,2]$. the $\left({ }^{3} \mathrm{He} / \mathrm{H}\right)_{B B N}$ error is mainly due to the $D\left({ }^{3} \mathrm{He}, p\right)^{4} \mathrm{He}$ process and, in a lower extent, to the $D(p, \gamma)^{3} \mathrm{He}$ reaction [1]. For what concern the ${ }^{7} \mathrm{Li}$ isotope, there are several reactions contributing to its abundance uncertainty. For $\eta \simeq 6 \cdot 10^{-10}$, the $\left({ }^{7} L i / H\right)_{B B N}$ error budget mainly come from the uncertainties of the ${ }^{7} \mathrm{Be}(\mathrm{n}, \alpha){ }^{4} \mathrm{He},{ }^{3} \mathrm{He}\left({ }^{4} \mathrm{He}, \gamma\right){ }^{7} \mathrm{Be}$ and ${ }^{7} \mathrm{Be}\left({ }^{2} \mathrm{H}, p\right) 2 \alpha$ reactions. Finally, the theoretical error of $\left({ }^{6} \mathrm{Li} / \mathrm{H}\right)_{p}$ is almost entirely due to the the $D(\alpha, \gamma)^{6} \mathrm{Li}$ process, that has been recently measured at BBN energies by the LUNA collaboration $[3,4,5]$.

The results of BBN theory have to be compared with the direct observations of light isotopes in

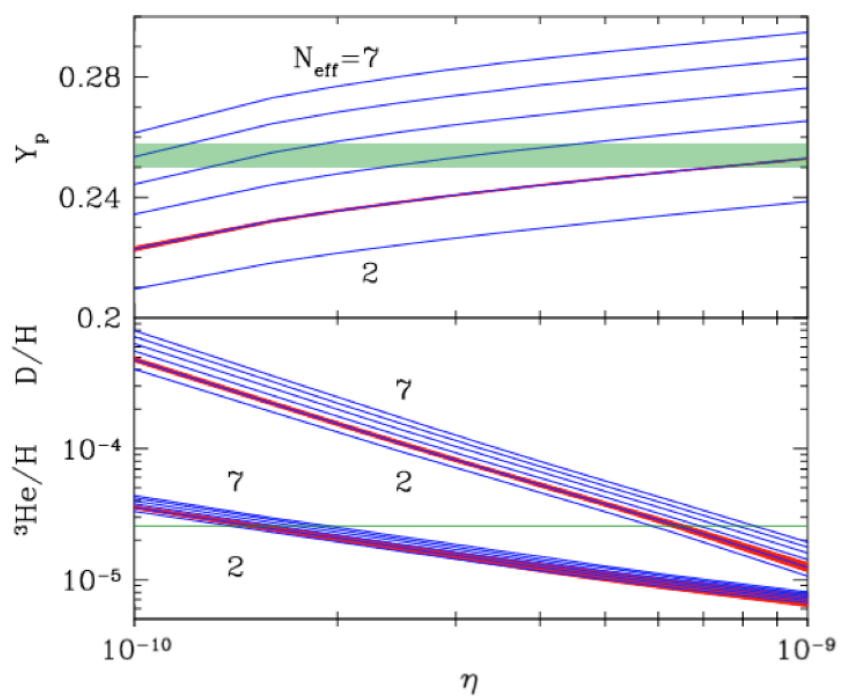

Figure 2: Yields of light nuclides as functions of the baryon-to-photon ratio, $\eta$. The blue lines indicate yields for a single value (integer plus 0.046) of $N_{\text {eff }}$. The red bands indicate the nuclear uncertainty on those yields for $N_{e f f}=3.046$. Also shown are horizontal green bands indicating observational constraints on ${ }^{4} \mathrm{He}$ and $D$ abundances [6,7].

the universe. As a general method, the primordial abundances are derived by extrapolating to zero metallicity the observations of light isotopes performed in metal-poor and/or faraway sites, where the abundances are though to be less polluted by processes as stellar burning and cosmic-ray interactions. The primitive abundance of ${ }^{4} \mathrm{He}$ is deduced from observations in HII (ionized hydrogen) regions of compact blue galaxies. The uncertainty is mainly due to systematics such as plasma temperature or stellar absorption [6]. Observations in Damped Lyman-Alpha (DLA) systems at high redshifts provide the primordial abundance of deuterium with good accuracy [7]. The ${ }^{3} \mathrm{He}$ observations are limited to our galaxy, and are affected by large systematics uncertainties because this isotopes is both produced and destroyed in stars so that its primordial amount is quite uncertain [8]. The lithium abundance at BBN epoch is deduced from the observations of its characteristic absorption line at about $680 \mathrm{~nm}$ in low metallicity stars in the galactic halo. The observations show that the lithium abundance is almost independent of metallicity ("Spite plateau" [9]). This constant abundance is interpreted as corresponding to the BBN lithium yield. Finally, it is reported in liter- 


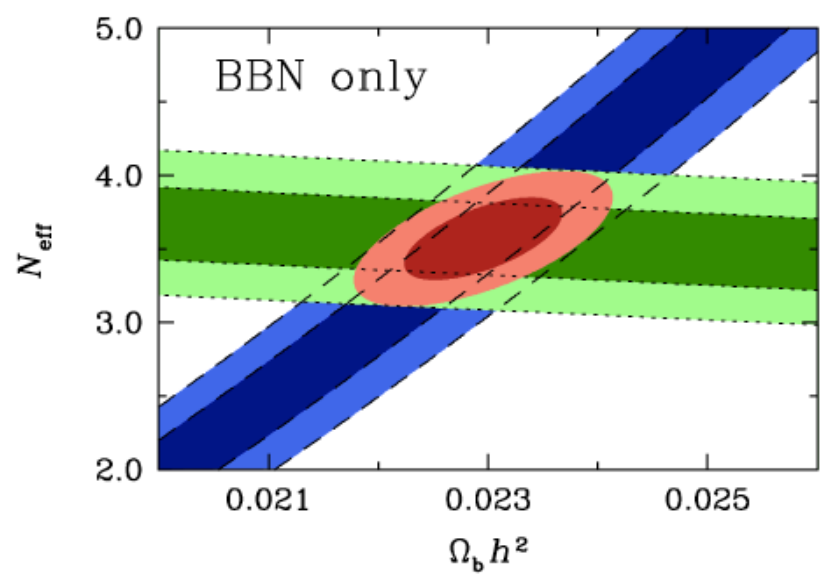

Figure 3: The $1 \sigma$ and $2 \sigma$ confidence contours (dark and light shades respectively) for $N_{\text {eff }}$ and $\Omega_{b, 0}$ derived from the primordial deuterium abundance (blue), the primordial He mass fraction (green), and the combined confidence contours (red) [7].

Table 1: Calculated and observed abundances of light isotopes derived from standard BBN and from direct astrophysical observations.

\begin{tabular}{lcc}
\hline \hline Isotope & SBBN Theory & Observations \\
\hline \hline$Y_{p}$ & $0.24771 \pm 0.00014[17]$ & $0.254 \pm 0.003[6]$ \\
$\mathrm{D} / \mathrm{H}$ & $(2.66 \pm 0.07) \times 10^{-5}[17]$ & $(2.53 \pm 0.04) \times 10^{-5}[7]$ \\
${ }^{3} \mathrm{He} / \mathrm{H}$ & $(1.00 \pm 0.07) \times 10^{-5}[33]$ & $(0.9 \div 1.3) \times 10^{-5}[8]$ \\
${ }^{7} \mathrm{Li} / \mathrm{H}$ & $\left(5.24_{-0.67}^{+0.71}\right) \times 10^{-10}[33]$ & $1.23_{-0.32}^{+0.68} \times 10^{-10}[34]$ \\
${ }^{6} \mathrm{Li} /{ }^{7} \mathrm{Li}$ & $(1.5 \pm 0.3) \times 10^{-5}[5]$ & ${ }^{6} \mathrm{Li} /{ }^{7} \mathrm{Li} \simeq 10^{-2}[24,11]$ \\
\hline
\end{tabular}

ature a controversial measurement in which the asymmetry of the absorption line of $L i$ is though to be due to the isotope shift in atomic lines of ${ }^{6} \mathrm{Li}$ with respect ${ }^{7} \mathrm{Li}[10,11]$.

Table 1 shows both the results of BBN calculations (assuming standard Model physics and the $\eta$ parameter derived from cosmic microwave background (CMB) experiments and the results of direct observations of light isotopes. The computed abundances of $\mathrm{D}$ and ${ }^{4} \mathrm{He}$ are in marginal agreement with observations, confirming the overall validity of the standard BBN theory. However, the tension between observations and theory may indicate the existence of dark radiation, as it will be discussed in the following. The ${ }^{3} \mathrm{He}$ is considered not a powerful probe for the BBN theory, because of the large systematics errors of the value derived from observations. For what concern the "heavy" isotopes of lithium, the abundance of ${ }^{7} \mathrm{Li}$ derived from observations $\left.\left({ }^{7} \mathrm{Li} / \mathrm{H}\right)_{o b s}\right)$ is a factor $\approx 4$ lower than the predicted $\left.{ }^{7} \mathrm{Li} / \mathrm{H}\right)_{p}$ ("The lithium problem") [12, 11]. Moreover, the amount of ${ }^{6} \mathrm{Li}$ observed in metal poor stars is unexpectedly large compared to BBN predictions $\left({ }^{6} L i_{o b s} /{ }^{6} L i_{B B N} \approx 10^{3}\right)$ [10]. Even though many of the claimed ${ }^{6} \mathrm{Li}$ detections are controversial, for a very few metal-poor stars there still seems to be a significant amount of ${ }^{6} \mathrm{Li}$ ("The second Lithium problem") $[12,11]$. The difference between predictions and observations of both the lithium isotopes may be due to new physics, such as the existence of supersimmetric particles in the early 
Universe $([12,11]$ and references therein).

In summary, the BBN theory provides a powerful tool to constrain particle physics and cosmology. A consistent theory (cosmology and particle physics) should provide the matching of BBN primordial abundances and the ones derived from observations. Therefore, it is presently very important a renewed study of the relevant nuclear processes, to reduce the error budget due to nuclear astrophysics. In the following I will focus the attention on the deuterium abundance, whose abundance depends to the baryon density and to the dark radiation. The study of the $D(p, \gamma)^{3} \mathrm{He}$ process at BBN energies is of crucial importance, as it is the main source of uncertainty of $(D / H)_{B B N}$, and $(D / H)_{o b s}$ is presently available with an error at the percent level. This study can be performed in existing [13] or in future underground accelerator facility

\section{Baryon density and neutrinos.}

The most recent CMB-derived baryon density is provided by the PLANCK collaboration [17]. Assuming standard model:

$$
\Omega_{b, 0}(C M B)=(2.205 \pm 0.028) / h^{2}
$$

In this equation, $\Omega_{b, 0}$ is the present day baryon density of the universe and $h$ is the Hubble constant in units of $100 \mathrm{~km} \mathrm{~s}^{-1} \mathrm{Mpc}^{-1}$.

The baryon density can be independently inferred by means of standard BBN theory, by comparing primordial deuterium abundance (by far, the most sensitive isotopes to $\Omega_{b, 0}$ ), with the value obtained with BBN calculations. The baryon density is independently obtained with comparison of $(D / H)_{B B N}$ and $(D / H)_{o b s}$. Observations in Damped Lyman-Alpha (DLA) systems at high redshifts provide the primordial abundance of deuterium $(D / H)_{p}=(2.53 \pm 0.04) \times 10^{-5}$, corresponding to the following Universal baryon density [7]:

$$
\Omega_{b, 0}(B B N)=(2.202 \pm 0.019 \pm 0.041) / h^{2}
$$

The error terms in eq. 2.2 reflect the uncertainties in observed deuterium abundance and BBN calculation [7]. The latter is due to the $3 \%$ uncertainty of computed $(D / H)_{B B N}$. In turn, this uncertainty is dominated by the experimental error of ${ }^{2} \mathrm{H}(p, \gamma)^{3} \mathrm{He}$ cross section at BBN energies $\left(30 \lesssim E_{c m}(k e V) \lesssim 400\right)[18,7]$. Therefore, a renewed accurate measurement of ${ }^{2} \mathrm{H}(p, \gamma)^{3} \mathrm{He}$ can remarkably reduce the error of the BBN-derived baryon density. It si worth to point out that the $\eta$ parameter obtained by $\mathrm{CMB}$ experiments and $\mathrm{BBN}$ theory gives respectively the Universal situation at $z \simeq 1000$ and $z \approx 10^{10}$. The matching of this independent measurements could represent a constrain to possible $\gamma$ annihilation of super symmetric particles generated in the early universe, after the first minutes of cosmic time and before the recombination epoch $(\simeq 380.000$ years).

In cosmology, the definition of "neutrino" is any relativistic particle contributing to the radiation density with respect to photons. For standard cosmology $N_{\text {eff }}=3.046$ and $\xi=0$, where $N_{\text {eff }}$ is the number of neutrino families and $\xi$ is the lepton asymmetry [7, 19]. Both $D$ and ${ }^{4} \mathrm{He}$ abundances depend on the expansion rate of universe, therefore they allow to bound $N_{\text {eff }}$ by using the BBN theory alone or in combination with CMB results [7, 18, 19]. Figure 2 shows the BBN abundance 


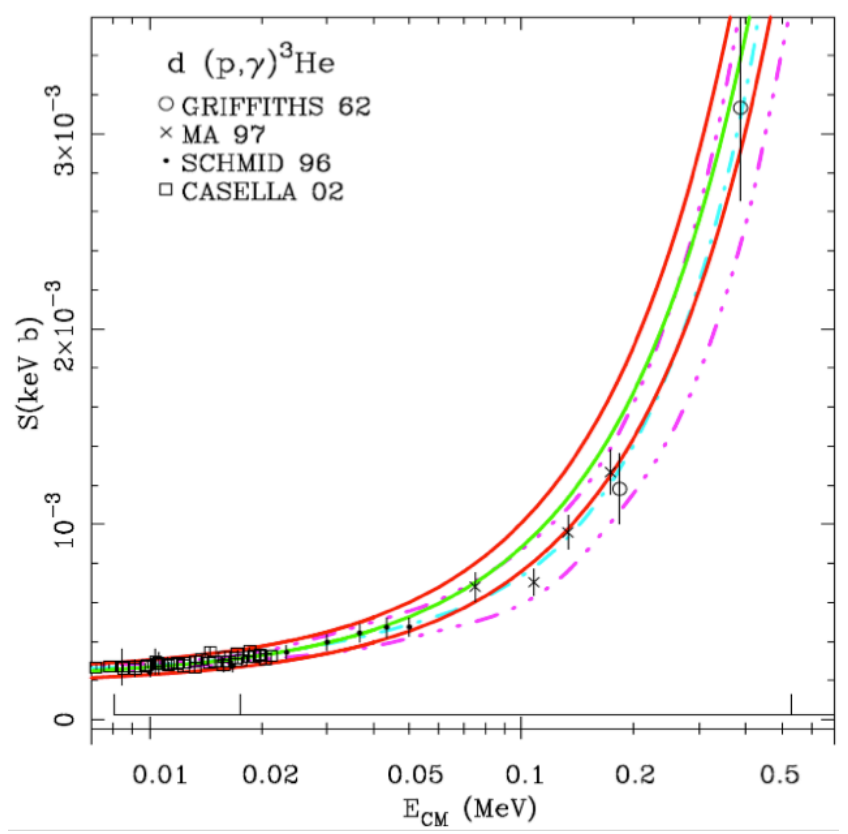

Figure 4: S-factor data for the reaction ${ }^{2} H(p, \gamma){ }^{3} H e$. The best-fit curve (dash-dot curves) and theoretical calculation (solid) are shown. All errors are shown as $2 \sigma \mathrm{s}$.

predictions as a function of the baryon-to-photon ratio, for $N_{e f f}=2$ to 4 . Also shown are the observational constraints on ${ }^{4} \mathrm{He}$ and $\mathrm{D}$ abundances.

The CMB-only bound is $N_{\text {eff }}(C M B)=3.36 \pm 0.34$ [17], while the BBN-only bound reported in [7] is $N_{e f f}(B B N)=3.57 \pm 0.18$. It is worthwhile to point out that both CMB and BBN constraints are in good agreement and provide a suggestive, but still inconclusive, hint of the presence of dark radiation. The BBN-only bound is graphically shown in Figure 3, in which the green band represents the confidence contour related to the helium abundance. As stated above (see 1), this uncertainty mainly depends on observational systematics. Instead, the blue band is the confidence contour for deuterium. According to eq. 2.2, this uncertainty mainly depends on the accuracy of the ${ }^{2} \mathrm{H}(p, \gamma)^{3} \mathrm{He}$ at BBN energy, that is the single most important obstacle to improve the BBN constraints on the existence of dark radiation.

\section{The deuterium abundance and $D(p, \gamma)^{3} \mathrm{He}$ reaction.}

In nuclear astrophysics the nuclear cross section $\sigma(E)$ is often factorized as follows:

$$
\sigma(E)=\frac{S(E) e^{-2 \pi \eta^{*}}}{E}
$$

$S(E)$ contains all the nuclear effects. For non-resonant reactions, it is a smoothly varying function of energy. In this formula, the exponential term takes into account the Coulomb barrier, while the astrophysical factor $S(E)$ contains all the nuclear effects. For non-resonant reactions, it is a smoothly varying function of energy. The Sommerfeld parameter $\eta^{*}$ is given by 
$2 \pi \eta^{*}=31.29 Z_{1} Z_{2}(\mu / E)^{1 / 2} . Z_{1}$ and $Z_{2}$ are the nuclear charges of the interacting nuclei. $\mu$ is their reduced mass (in units of a.m.u.), and $E$ is the center of mass energy (in units of $k e V$ ).

Figure 4 shows the available $S_{12}$ data for this reaction. The precise low-energy data come from the LUNA measurement performed with the $50 \mathrm{kV}$ accelerator [20]. Only a single dataset of $S_{12}$ is currently available in the relevant energy range [21], in which the authors state systematic uncertainty of $9 \%$. Figure 4 also shows the energy dependence of $S_{12}$ as obtained by "ab initio" calculations $[18,22,23,24]$.
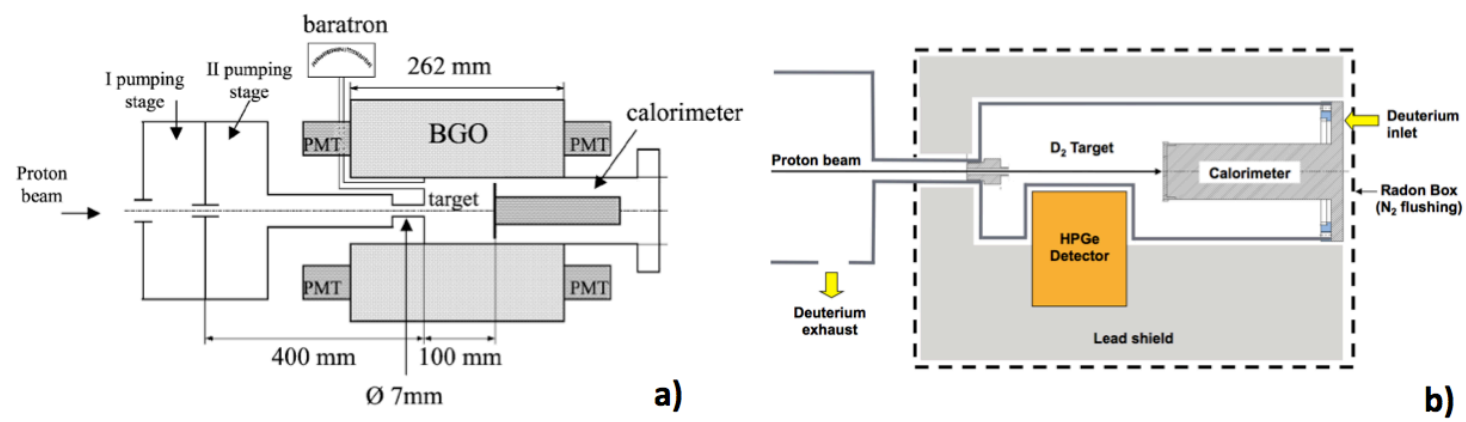

Figure 5: a): Scheme of gas target setup and BGO detector. b): Scheme of gas target setup and HPGe detector.

The feasibility of studying the ${ }^{2} \mathrm{H}(\mathrm{p}, \gamma)^{3} \mathrm{He}$ reaction $(Q=5.5 \mathrm{MeV})$ with good accuracy has been demonstrated at $2.5<E_{c m}(\mathrm{keV})<22$ with the previous LUNA $50 \mathrm{kV}$ accelerator (see figure 4) [20]. Figure 5 a) shows the scheme of the setup used in [20], where a barrel BGO detector is implemented. Figure $5 \mathrm{~b}$ ) shows the scheme of the setup used for the study of the ${ }^{2} H(\alpha, \gamma){ }^{6} \mathrm{Li}$ reaction, where a large Germanium (HPGe) detector detects the photons produced in the reaction [25]. The high efficiency ( $\sim 70 \%$, see [20]) of the LUNA BGO reduces the dependence of the detector response on the angular distribution of the emitted $\gamma$ rays and thus is a prerequisite to achieve a low systematic uncertainty. The detection efficiency can be determined by precise Monte Carlo simulations, as well as performing dedicated measurements and calibrations, e.g. by measuring the absolute efficiency at a $\gamma$ ray energy of $6.13 \mathrm{MeV}$ exploiting the $340 \mathrm{keV}$ resonance in the ${ }^{19} \mathrm{~F}(p, \alpha \gamma){ }^{16} \mathrm{O}$ reaction. With the proposed setup the expected counting rate (full detection $\gamma$-peak) is of the order of $10^{4}-10^{5}$ events/hour in the considered energy range (see figure 6), making the measurements with BGO detector relatively fast for what concern statistics and allowing to determine the beam heating effect and the target density in asymptotical conditions, by performing dedicated measurements in which target pressure and beam intensity are varied. Finally, the beam intensity error can be minimized by a proper calibration of the calorimeter (1.5\% uncertainty in ref. [26]). Although the large angular coverage of BGO detector makes the counting yield nearly independent of the angular distribution of emitted photons, an exhaustive study of the ${ }^{2} \mathrm{H}(p, \gamma)^{3} \mathrm{He}$ reaction includes the study of angular distribution of emitted $\gamma$-rays, in order to precisely evaluate the response of BGO detector. This study can be accomplished by using the HPGe detector facing the gas target in a close geometry, as it is shown in figure 5b). The angular distribution can be 
inferred by exploiting the high energy resolution of the detector and the doppler effect affecting the energy of $\gamma$ 's produced along the beam line by the ${ }^{2} H(p, \gamma)^{3} \mathrm{He}$ reaction. This study provides a useful tool for nuclear theoretical models. In fact, as stated above, the tension between existing data at BBN energies and "ab initio" calculations is presently debated.

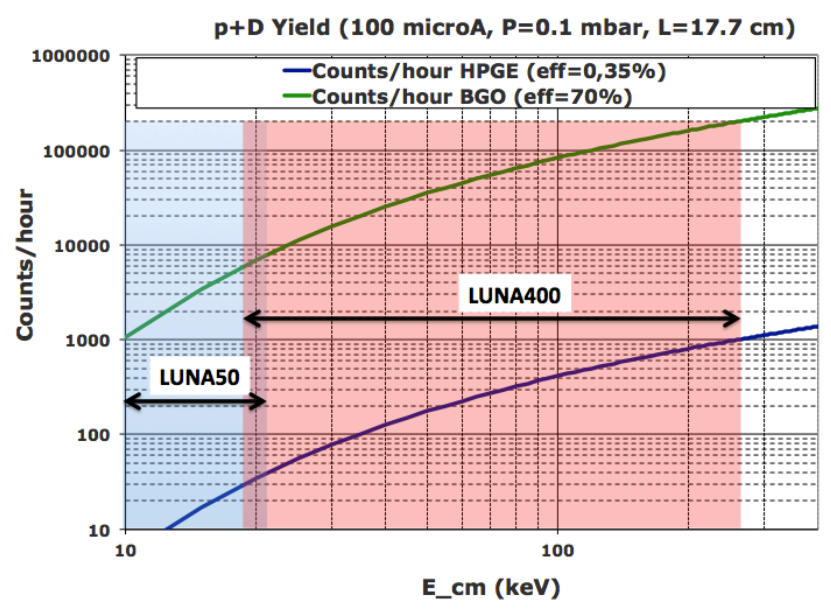

Figure 6: Reaction Yields at LUNA400, using the BGO detector (green line, see figure 5a) and the HPGe detector (blue line, see figure 5b). In the figure the working conditions, the energy range of the proposed experiment and the one of the previous LUNA measurement are also indicated [20].

\section{Conclusions}

The progress on direct observations of deuterium abundance [7] and the accuracy of CMB data [17] make the lack of ${ }^{2} \mathrm{H}(\mathrm{p}, \gamma)^{3} \mathrm{He}$ reaction data at $\mathrm{BBN}$ energies the main obstacle to improve the constraints on $\Omega_{b, 0}(B B N), N_{e f f}$ and lepton degeneracy $\xi[7,18,27,28]$. A precision study of the ${ }^{2} H(p, \gamma)^{3} \mathrm{He}$ reaction inside the BBN energy region of interest, with the goal of improving the present $9 \%$ systematic uncertainty of the $S_{12}$ factor, is extremely important in this concern. As light nuclei are involved in this process, the ${ }^{2} \mathrm{H}(p, \gamma)^{3} \mathrm{He}$ reaction is of high interest also in theoretical nuclear physics, in particular for what concern "ab-initio" modelling. To reduce the background induced by cosmic rays, this study must be performed with facilities operating underground. Presently, the only underground accelerator in the world is the LUNA $400 \mathrm{kV}$ accelerator at the Gran Sasso laboratory, Italy [13]. The "Gran Sasso" mountain provides a natural shielding which reduces the muon and neutron fluxes by a factor $10^{6}$ and $10^{3}$, respectively. The suppression of the cosmic ray induced background also allows an effective suppression of the $\gamma$-ray activity by a factor $10^{2}-10^{5}$, depending on the photon energy [29]. The ultra-low background at LNGS made possible the study of other leading processes of the BBN network, such as ${ }^{2} \mathrm{H}(p, \gamma)^{3} \mathrm{He}$ and ${ }^{2} \mathrm{H}\left({ }^{3} \mathrm{He}, p\right){ }^{4} \mathrm{He}$ at very low energy [20,30,31], by using the $50 \mathrm{kV}$ accelerator [32], and the ${ }^{3} \mathrm{H}\left({ }^{4} \mathrm{He}, \gamma\right){ }^{7} \mathrm{Be}$, already studied with the present facility [13]. The low environmental background achievable underground made possible the recent first direct measurement of the ${ }^{2} H(\alpha, \gamma){ }^{6} \mathrm{Li}$ astrophysical factor at Big Bang energies [5, 3, 4], providing a solid experimental footing to calculate 
the $\left({ }^{6} \mathrm{Li} / \mathrm{H}\right)_{B B N}$ primordial abundance. More in general, the present "Era of precision cosmology" requires the accurate measurements of several reaction at BBN energies, in the whole BBN energy range. This program can be accomplished with present (LUNA) and proposed facilities $[14,15,16]$.

\section{References}

[1] P.D. Serpico et al., J. Cosmol. Astropart. Phys. 2004, 010 (2004).

[2] D.S. Leonard et al., Phys. Rev. C 73045801 (2006).

[3] C. Gustavino: "LUNA data on the ${ }^{2} H(\alpha, \gamma)^{6} L i$ reaction and the Big Bang Nucleosynthesis", PoS(NIC XII)074 (2012).

[4] C. Gustavino: "The ${ }^{2} H(\alpha, \gamma)^{6} L i$ experiment at LUNA", EPJ Web of Conferences Volume 66, 2014.

[5] M. Anders et al.: Phys. Rev. Lett., submitted.

[6] Y.I Izotov, G. Stasinska, N.G. Guseva, arXiv:1308.2100 (2013).

[7] R.J. Cooke and M. Pettini: arXiv:1308.3240v1 [astro-ph.CO] 14 Aug 2013.

[8] T. Bania, R. Rood and D. Balser Nature 415, 54 (2002).

[9] M. Spite and F. Spite, Nature 297, 483 (1982).

[10] M. Asplund, D.L. Lambert, P.E. Nissen, F. Primas and V.V. Smith, ApJ, 644, 229 (2006).

[11] See proceedings of "Lithium in the Cosmos", 27-29 February 2012, Paris.

[12] B.D. Fields, Annu. Rev. Nucl. Part. Sci. 61, 47 (2011).

[13] A. Formicola et al., Nucl. Instr. and Meth. A 507 (2003) 609.

[14] M. Junker: "Experiences and Prospects of Nuclear Astrophysics in Underground Laboratories", proceedings of "Nuclear Physics in Astrophysics VI", 19-24 May 2013, Lisbon.

[15] D. Bemmerer: "5 MeV underground accelerator in the Dresden Felsenkeller", proceedings of "Nuclear Physics in Astrophysics VI", 19-24 May 2013, Lisbon.

[16] A. Lemut: "The DIANA Underground Accelerator Facility", proceedings of "Nuclear Physics in Astrophysics VI", 19-24 May 2013, Lisbon.

[17] PLANCK collaboration: arXiv:1303.5076v1 [astro-ph.CO] 20 Mar 2013.

[18] K.M. Nollett and G.P. Holder: arXiv:1112.2683v1 [astro-ph.CO] 12 Dec 2011.

[19] G. Steigman: arXiv:1208.0032v1 [hep-ph] 31 Jul 2012.

[20] C. Casella et al.: Nuclear Physics A 706 (2002) 203Đ216.

[21] L. Ma et al., Phys. Rev. C 55, 588 (1997).

[22] M. Viviani et al., Phys. Rev. C 61, 064001 (2000), arXiv:nucl-th/9911051.

[23] L. E. Marcucci et al., Phys. Rev. C 72, 014001 (2005), arXiv:nucl-th/0502048.

[24] A. Kievsky et al., Journal of Physics G Nuclear Physics 35, 063101 (2008), arXiv:0805.4688.

[25] M. Anders et al.: Eur. Phys. J. A (2013) 49: 28.

[26] H. Costantini et al.: Nuclear Physics A 814 (2008) 144Đ158. 
[27] A. Melchiorri (PLANCK Collaboration), private communication.

[28] G. Miele (PArthENoPE code), private communication.

[29] A. Caciolli et al., Eur. Phys. J. A 39, 179 - 186 (2009).

[30] H. Costantini et al., Phys. Lett. B 482, $43 Đ 49$ (2000).

[31] M. Aliotta et al., Nucl. Phys. A 690, $790 Đ 800$ (2001).

[32] U. Greife et al, NIM A 350 (1994). Volume 350, 327Đ337, (1994).

[33] R.H. Cyburt, B.D. Fields and K.A. Olive JCAP 11, 12 (2008).

[34] S.G. Ryan, et al. Astrophys. J. Lett. 530:L57 (2000). 